Cite this: Analyst, 2013, 138, 4649

\title{
A new quantitative method for gunshot residue analysis by ion beam analysis $\uparrow$
}

\author{
Matthew E. Christopher, ${ }^{a}$ John-William Warmenhoeven, ${ }^{\text {ab }}$ Francesco S. Romolo, de \\ Matteo Donghi, ${ }^{\mathrm{c}}$ Roger P. Webb, ${ }^{a}$ Christopher Jeynes, ${ }^{a}$ Neil I. Ward, ${ }^{\mathrm{b}}$ Karen J. Kirkby ${ }^{\mathrm{a}}$ \\ and Melanie J. Bailey ${ }^{* b}$ \\ Imaging and analyzing gunshot residue (GSR) particles using the scanning electron microscope equipped \\ with an energy dispersive X-ray spectrometer (SEM-EDS) is a standard technique that can provide \\ important forensic evidence, but the discrimination power of this technique is limited due to low \\ sensitivity to trace elements and difficulties in obtaining quantitative results from small particles. A new, \\ faster method using a scanning proton microbeam and Particle Induced X-ray Emission ( $\mu$-PIXE), \\ together with Elastic Backscattering Spectrometry (EBS) is presented for the non-destructive, \\ quantitative analysis of the elemental composition of single GSR particles. In this study, the GSR particles \\ were all $\mathrm{Pb}, \mathrm{Ba}$, Sb. The precision of the method is assessed. The grouping behaviour of different makes \\ of ammunition is determined using multivariate analysis. The protocol correctly groups the cartridges \\ studied here, with a confidence $>99 \%$, irrespective of the firearm or population of particles selected.
}

Received 27th March 2013 Accepted 4th June 2013

DOI: $10.1039 / c 3 a n 00597 f$

www.rsc.org/analyst
(ASTM) in 2010 states that particles which are "characteristic of GSR" must contain lead, barium and antimony. Such particles may contain one or more of the following elements: aluminum, silicon, phosphorus, sulphur (trace), chlorine, potassium, calcium, iron (trace), nickel, copper, zinc, zirconium and tin. ${ }^{5}$ The morphology and the elemental analysis of recovered particles can be interpreted following a formal approach which allows them to be judged as "characteristic" or "consistent" with GSR. For more meaningful interpretation of GSR particles "the recovered particulate can be compared with case-specific known source items, such as the recovered weapon, cartridge case or victim related items".,5,5

Scanning Electron Microscopy with Energy Dispersive X-ray Spectrometry (SEM-EDS) has become the technique of choice for GSR analysis due to the fact that it is widely available, spatially discriminating and non-destructive., ${ }^{2,6,7}$ When cartridge cases of various types of ammunition are recovered from the crime scene, the comparison of GSR particles from the known sources with those found on a suspect can sometimes result in inconclusive discussions in court, because of the poor sensitivity of the technique to trace elements and the inability to provide quantitative information from single particles. ${ }^{8,9}$ There is, therefore, an urgent need for a technique that retains this non-destructive nature, yet has a greater capability than SEM-EDS to collect chemical information (including quantitative information) from single GSR particles, allowing for greater discrimination between different sources.

Of the techniques that have been previously tested for the analysis of GSR particles, Laser Ablation Inductively Coupled Plasma-Mass Spectroscopy (LA-ICP-MS) offers detection limits 
in the $\mu \mathrm{g} \mathrm{kg}^{-1}$ to $\mathrm{mg} \mathrm{kg}{ }^{-1}$ (ppb-ppm) range for most elements, ${ }^{10}$ but lacks the spatial resolution to locate and image GSR particles, which are typically a few microns in size amongst the large population of environmental particles that are normally collected from a suspect. Additionally, the technique is destructive, making it unsuitable for casework, in which only a small number of particles are typically recovered. ${ }^{11}$ Time-ofFlight Secondary Ion Mass Spectrometry (ToF-SIMS) is capable of analyzing individual GSR particles with a good sensitivity for both organic and inorganic molecules. However, due to the surface sensitive nature of the technique, sputtering of the particles is required in order to expose the area of interest, making it quasi-destructive. ${ }^{12}$ ToF-SIMS additionally suffers from difficulties in producing quantitative measurements, particularly for GSR samples with non-uniform topography. ${ }^{\mathbf{1 3}}$ $\mathrm{X}$-Ray Fluorescence (XRF) ${ }^{\mathbf{1 4}}$ and Raman spectroscopy ${ }^{15}$ are also non-destructive but lack spatial resolution and also suffer from quantification problems. SEM with Wavelength Dispersive X-ray Spectrometry (SEM-WDS) ${ }^{16}$ and SEM with Transition Edge Sensor (SEM-TES) using a microcalorimeter detector ${ }^{17}$ offer enhanced sensitivity to trace elements compared with SEM-EDS but are unable to provide quantitative information for this type of sample.

The Ion Beam Analysis (IBA) scanning microbeam technique Particle Induced X-ray Emission ( $\mu$-PIXE) uses a beam of protons with energy between 2 and $3 \mathrm{MeV}$ to produce the same $\mathrm{X}$-ray emissions as in SEM-EDS, but $\mu$-PIXE detects elements at the $\mathrm{mg} \mathrm{kg}^{-1}$ (ppm) level due to the much lower primary bremsstrahlung background produced by the protons (compared to the electrons in the SEM). Previous preliminary work by Bailey et al. has shown that $\mu$-PIXE therefore has a higher sensitivity than SEM-EDS to the trace elements in GSR particles. ${ }^{18,19}$ Romolo et al. ${ }^{20}$ have recently demonstrated that $\mu$-PIXE together with Particle Induced Gamma Emission (PIGE) is also able to give unprecedented characterization of GSR particles arising from primers not containing lead.

In SEM-EDS, backscattered electron energy spectra are almost invariably treated qualitatively due to the predominance of multiple scattering effects within the sample. ${ }^{21}$ In contrast, backscattered particles from the ion beam usually undergo only one scattering event, encouraging quantitative interpretation of the particle energy spectrum. The particle spectrum (EBS) spectrum allows the calculation of total charge deposited and the variation in the matrix signal as a function of depth. ${ }^{22-24}$ This in turn allows the X-ray absorption to be accurately calculated, enabling quantitative analysis of the constituent elements. Bailey et al. ${ }^{18,19}$ reported preliminary quantitative data from GSR particles obtained from self-consistent treatment of PIXE and EBS data, which was found to be too time consuming for use on large populations of particles. Romolo et al. ${ }^{20}$ reported data on primers not containing lead, using a more simplified analytical procedure but requiring elemental mapping, which is also too time consuming for large populations of particles.

The present paper describes a significant improvement to the above analytical procedures to allow effective characterisation of GSR particle populations. For the construction of databases, where a very large number of particles and corresponding spectra would necessarily be studied, a streamlined analysis procedure is required. This is developed and tested in this work where we present a new method for GSR analysis using IBA and multivariate analysis, giving, for the first time, the capability to obtain and rapidly evaluate quantitative elemental information relating to single GSR particles. This is used to characterise and compare populations of GSR particles for forensic purposes, with the opportunity to improve the support from chemical information to criminal investigations involving firearms.

\section{Materials and methods}

\subsection{Sample collection}

GSR samples were collected from various different sources, as shown in Table 1. The cartridge makes studied here were selected because they are known to generate "characteristic" particles containing $\mathrm{Pb}, \mathrm{Ba}$ and $\mathrm{Sb} .{ }^{5}$ Residues from the hands of shooters were collected by dabbing their hands 100 times with SEM stubs covered with Leit adhesive carbon tabs (supplied by Agar Scientific), concentrating on the top of the thumb and index finger. ${ }^{25}$ Residues from spent cartridge cases were obtained by scraping the inside with swabs before transferring the residue to the stubs. Shooters' hands were thoroughly washed between each firing and dried on disposable paper towels. A blank collection was taken between hand washing and firing. In subsequent analysis, 1 particle of GSR was found on 2 of the blank stubs. This was not considered to be a problem as the majority of results presented here are based on populations of between 14 and 30 particles per firing. Fresh latex gloves were used for each collection and sampling was carried out over 10 meters away from the firing site in order to avoid any airborne contamination of the stubs.

\subsection{Particle relocation protocol and ion beam analysis conditions}

To test the relocation abilities of this method, the positions of 11 GSR particles of sizes varying from $60 \mu \mathrm{m}$ to $1 \mu \mathrm{m}$ in diameter (including three of $1 \mu \mathrm{m}$ and two of respectively $3 \mu \mathrm{m}$ and $5 \mu \mathrm{m}$ ) were located using a FEI Quanta 400 SEM, equipped with an EDAX Phoenix EDS system and E.A. GSR XT software at the GSR laboratory of Reparto Investigazioni Scientifiche (RIS) Carabinieri in Parma. The positions were logged relative to indents created with a scalpel in the four cardinal directions on the edge of the stub with 'north' being a triple indent. The remaining SEM stubs were covered with a copper particle finder grid supplied by Gilder Grids. Linconshire, UK (reference code SEMF3). A Philips XL30 scanning electron microscope fitted with an EDAX "EDX Control" system was used to locate and log the positions of individual GSR particles of approximately spherical geometry containing $\mathrm{Pb}, \mathrm{Ba}$ and $\mathrm{Sb}$ at the University of Surrey Advanced Technology Institute. The beam energy was $30 \mathrm{keV}$ and the detector was a $133 \mathrm{eV} \mathrm{Si(Li)} \mathrm{UTW} \mathrm{(ultra-thin}$ window) detector with a take-off angle of $45^{\circ}$ and energy resolution of $133 \mathrm{eV}$ at $\mathrm{Mn} \mathrm{K \alpha}$. 
Table 1 Details of each sample used in this work

\begin{tabular}{|c|c|c|c|c|c|c|}
\hline Sample no. & $\begin{array}{l}\text { No. of } \\
\text { particles }\end{array}$ & Sample label & Source & Ammunition name & Firearm & $\begin{array}{l}\text { Beam current and } \\
\text { spot size }\end{array}$ \\
\hline 1 & 18 & SP1 Re-run & Hands & Federal $9 \mathrm{~mm}$ Luger & SIG Sauer P226 & $600 \mathrm{pA}, 3.5 \times 4 \mu \mathrm{m}$ \\
\hline 2 & 14 & SP1 & Hands & & SIG Sauer P226 & $750 \mathrm{pA}, 2 \times 3 \mu \mathrm{m}$ \\
\hline 3 & 19 & SP2 & Hands & & SIG Sauer SP2022 & \\
\hline 4 & 18 & SP3 & Hands & & Glock 17 & $800 \mathrm{pA}, 3 \times 3 \mu \mathrm{m}$ \\
\hline 7 & 5 & MAG2 & Hands & Federal $0.357^{\prime \prime}$ Magnum & Smith \& Wesson 686 & $500 \mathrm{pA}, 3 \times 3 \mu \mathrm{m}$ \\
\hline 8 & 20 & HYD1 & Hands & Federal 9 mm Luger 124 grain & Glock 19 & $500 \mathrm{pA}, 3 \times 3 \mu \mathrm{m}$ \\
\hline 9 & 20 & HYD2 & Hands & Hydra-Shock Jacketed Hollow Point & & \\
\hline
\end{tabular}

Relocation of particles in the ion beam was carried out using several ion-beam-induced secondary electron images at increasing magnification, together with the previously logged distances and SEM images to ascertain the general area of interest. For the highest magnification images, elemental X-ray maps (typically taking 1-2 minutes to generate) were used to find the exact location of each of the smallest particles. $\mu$-PIXE spectra were generated using a $3.0 \mathrm{MeV}$ proton beam with beam currents of 500-800 pA, focussed to spot sizes between $1 \mu \mathrm{m}$ and $4.5 \mu \mathrm{m}$ (see Table 1), and a $146 \mathrm{eV} \mathrm{Si}(\mathrm{Li})$ detector at a take-off angle of $45^{\circ}$ with a $130 \mu \mathrm{m}$ be filter to exclude backscattered protons from the detector. Backscattered particle (EBS) spectra were simultaneously collected at a scattering angle of $155^{\circ}$ and a solid angle of $50 \mathrm{~m}$ sr. Energy calibration of $\mu$-PIXE and EBS spectra was performed using the Pb-glass BCR-126A standard from the Institute for Reference Materials and Measurement, Geel, Belgium.

\subsection{Spectral quantification and fitting protocol}

A point analysis (typically 5 minutes per analysis) in the centre of each particle was obtained. The OMDAQ ${ }^{24}$ software, which has its own EBS code and implements GUPIX ${ }^{26}$ for the analysis of PIXE spectra, was used to fit the IBA spectra (PIXE and EBS) self-consistently, using an approximate initial composition of a metal dioxide $(\mathrm{Pb} 0.2$, $\mathrm{Ba} \mathrm{0.5}, \mathrm{Sb} \mathrm{0.2,} \mathrm{S} 0.05$ and $\mathrm{O} 2.0)$ for the particle and a carbon tape substrate with $\mathrm{C} / \mathrm{O}=8$ (from measurements of a blank area of the carbon stub). The composition and thickness of the particle layer was then automatically obtained by fitting the data, with SEM images used as a check of the thickness obtained, ensuring a representative matrix had been used in the fitting procedure. This process takes around 2 minutes per particle.

\subsection{Background subtraction protocol}

Due to the greater sampling depth of the ion beam compared with the SEM (tens of microns compared with a few microns) it is possible to generate $\mathrm{X}$-rays from the adhesive layer, which varies in composition across the substrate, depending on the amount of glue present. To account for this, background information spectra were collected from the blank spots on every stub used, varying between heavy glue presence and none. We have already shown how to deal with background in quantitative analysis using elemental maps, ${ }^{20}$ but for larger populations of particles acquiring maps is too time consuming. Therefore, in the present research, this set of 16 measurements was used to produce a theoretical 'worst case' stub by amalgamating the largest concentration of each element detected in each measurement of all the blank stubs (see ESI $\dagger$ ). The resulting background is quantified explicitly in the ESI (Fig. S1†). The worst case stub concentration was then subtracted from the calculated concentration of each element detected in each particle to ensure that the reported element comes from the particle and not the substrate. The minimum detection limits relevant here (of order 100 times higher than typical PIXE measurements) are then given by these "blank" values. These poor detection limits are only an inconvenience since, as described later, only the major and minor elements were found to be significant in separating the populations of particles.

\subsection{Analysis protocol}

The background-subtracted quantitative elemental content data were then entered into a statistical analysis program, IBM SPSS Statistics. ${ }^{27}$ Multivariate canonical discrimination function analysis (CDFA) was employed in order to test how well the different makes of ammunition could be separated. The Wilks' lambda distribution was used to evaluate the statistical significance of the separation.

\section{Results}

\subsection{Damage test}

The non-destructive nature of IBA was tested by leaving a 2.5 $\mathrm{MeV}$ beam focused to $4.5 \mu \mathrm{m} \times 5 \mu \mathrm{m}$ with a beam current of $3 \mathrm{nA}$ (considerably greater than a typical analysis), for a 850 minutes point analysis on one of GSR particles from a Federal $9 \mathrm{~mm}$ Luger 95 grain Jacketed Soft Point cartridge. SEM images of the particle before and after analysis are shown in Fig. 1 along with an overlay of the $\mu$-PIXE spectra for the first and last $5 \%$ of the data acquired. PIXE spectra were also fitted for every $10 \%$ of the data collected, with relative standard deviations (RSDs) at 

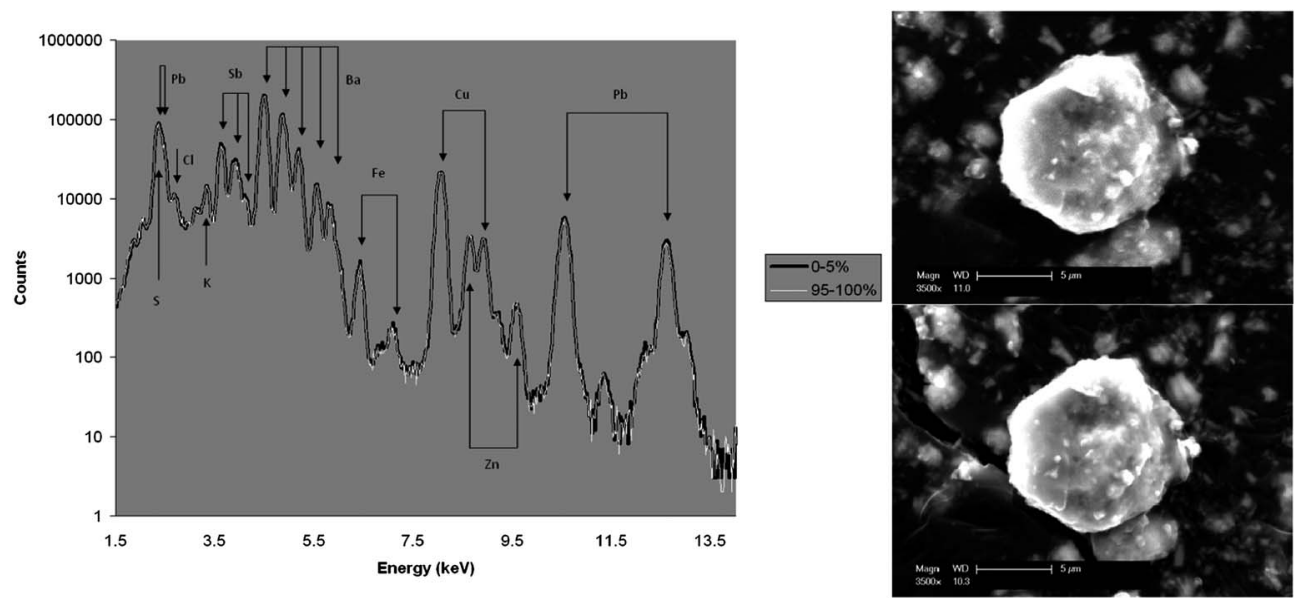

Fig. 1 SEM images of a particle of GSR originating from Federal $9 \mathrm{~mm}$ Luger 95 grain Jacketed Soft Point before (above) and after (below) overnight analysis. Overlay (left) of PIXE spectra corresponding to the first and last $5 \%$ of the data.

levels (of between 0.6 and $5.6 \%$ for all elements detected) expected from the counting statistics. No morphological changes were observed by SEM analysis, even under this unrealistically long exposure time, indicating that the sample was not measurably compromised, making reanalysis possible.

\subsection{Relocation}

In the procedure described above under the 'Relocation Protocol' it was possible to relocate 9 out of the 11 test particles with the smallest being $\sim 1 \mu \mathrm{m}$ and the missing particles both being $1 \mu \mathrm{m}$ or less in diameter. The capability to find $1 \mu \mathrm{m}$ particles depended on a well-focused beam $<2 \mu \mathrm{m}$.

\subsection{Precision, homogeneity and reproducibility}

Samples 1 and 2 are repeat measurements of the same particles on dates four months apart. Fig. 2 illustrates box plots of the distribution of the RSD between the measurements for each element detected, excluding $\mathrm{Zn}$ and $\mathrm{Cl}$ which were only detected in one and two particles respectively. The RSDs are typically less than $10 \%$ for most elements; $\mathrm{Cu}, \mathrm{K}$ and $\mathrm{P}$ being the only exceptions. This is because $\mathrm{K}$ and $\mathrm{P}$ were both detected in

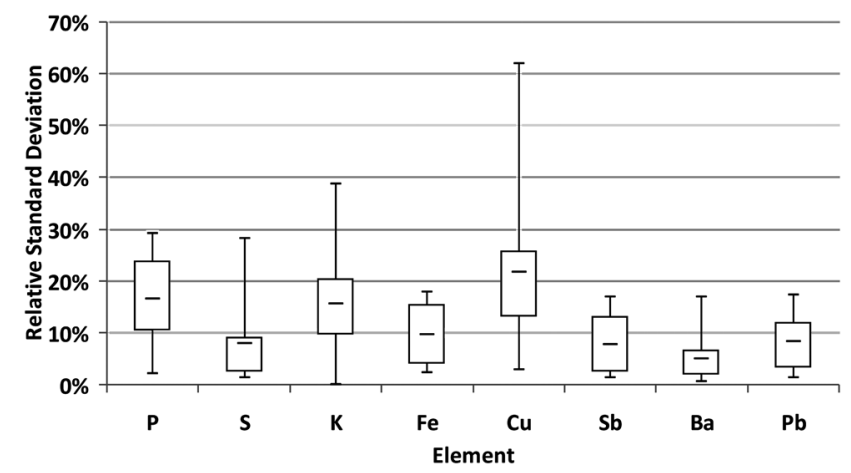

Fig. 2 Box plots of the relative standard deviation between the concentrations of the elements detected when the same group of 10 particles were analysed on dates 4 months apart. concentrations very close to the limit of detection and $\mathrm{Cu}$ was found to be spatially inhomogenous from mapping of GSR particles.

\subsection{Intra-box variability}

Samples 8 and 9 are sets of particles produced by different cartridges from the same box of ammunition with the same firearm. The particles were correctly grouped together using CDFA and are separated from the other two types of cartridges. The function employed to discriminate the residues returned a Wilks' Lambda value of 0.675 with a significance below the $95 \%$ confidence level ( $p=0.154)$, demonstrating that these residues, coming from a similar source, could not be discriminated. Box plots (see ESI $\dagger$ ) showed no significant difference between the samples for any of the elements detected.

\subsection{Effect of changing the type of firearm}

GSR particles have a complex genesis and a memory effect due to different cartridges previously shot by the same firearm has been reported. ${ }^{28}$ We have also investigated this.

In samples 2 to 6 there are particles produced by ammunition taken from the same box, but shot by 5 different firearms. Using CDFA, the samples could not be discriminated. The 4 functions employed to discriminate the residues returned Wilks' lambda values of 0.659 with a significance below the $95 \%$ confidence level $(p=0.102)$, demonstrating that these residues, coming from a similar source, could not be discriminated. Box plots (see ESI $\dagger$ ) showed no significant difference between the samples for any of the elements detected.

\subsection{Variability between three different makes of ammunition}

Fig. 3 plots the first two canonical discriminant functions of all the samples. The dark boxes represent the peak centroids of the data for each sample and show discrimination between the 3 different makes of ammunition. Samples 1-6 are residues formed by a first make of ammunition (Make 1 is Federal $9 \mathrm{~mm}$ Luger 


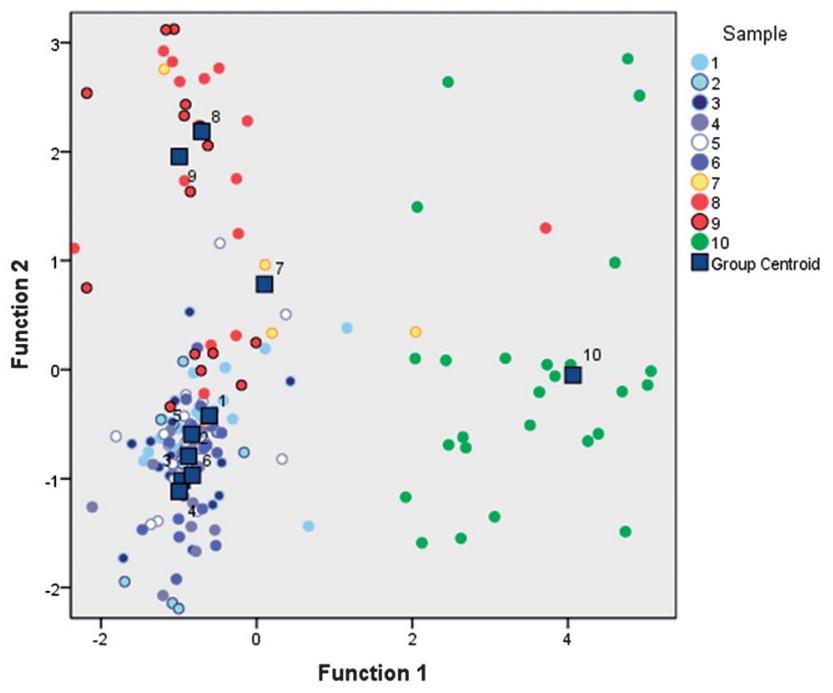

Fig. 3 Canonical discriminant function graphs showing clear discrimination between each of the three makes of Federal ammunition (Make $1=$ samples 1-6; Make 2 = sample 7; Make 3 = sample 8-9) sampled from hands as well as the sample from a cartridge case of Make 1 (sample 10).

95 grain Jacketed Soft Point) collected from hands, Sample 10 is residue from the cartridge case of this Make 1, Sample 7 is residue from a second make (Make 2 is Federal $0.357^{\prime \prime}$ Magnum) collected from hands and samples 8-9 are residues from a third make (Make 3 is Federal 9 mm Luger 124 grain Hydra-Shock Jacketed Hollow Point) collected from hands. Three functions were used to separate the data and explained $62.8 \%, 18.0 \%$ and $11.1 \%$ of the variance respectively, returning Wilks' Lambda values of 0.072 with a significance of $0.000(p=0.000)$; the separation therefore being significant past $99 \%$ confidence. The degree of success with which the model identified the make of ammunition was determined by calculating the percentage of cases that were correctly classified, known as the hit ratio. This was $91 \%$ (86\% using a cross-validated algorithm). Box plots showing the statistical distribution of the concentration of elements detected in the population of particles from each sample are presented in the ESI. $\uparrow$ These give support to the separation achieved by the CDFA analyses, showing that Make 1 is characterised by low $\mathrm{Cu}$; Make 2 is characterised by low $\mathrm{S}, \mathrm{Ba}$ and $\mathrm{Sb}$, with a number of particles containing $\mathrm{Ni}$; and that Make 3 is characterised by high $\mathrm{Cl}, \mathrm{Cr}$ and Fe.

A potential limitation of the protocol is a systematic error introduced by the background subtraction of the worst case stub. The effect of this method on the ability to group the makes of cartridges was studied and CDFA results of data without background subtraction are presented in the ESI, $\uparrow$ showing that the grouping behaviour is unaffected. The background subtraction method is necessary as it reduces the risk of falsely identifying an element as being present in a GSR particle. This is important in ensuring the compatibility of this protocol with the qualitative assessment of GSR particle composition that is currently used in casework.

\subsection{Effect of sample size}

The effect of sample size on the ability to associate GSRs with a reference population was tested using sample 8 as the 'unknown' ammunition and sample 9 as the reference population, both samples originating from the same ammunition. The entire dataset for all 3 makes of ammunition was entered into SPSS, excluding that of sample 8, from which groups of particles were randomly selected before CDFA was used to classify them. Fig. 4 presents some of the results from this test with graphs for the secondary group containing 10, 8, 5 and 3 particles going from left to right. The correlation between equivalent samples becomes increasingly poor as the size of sample 8 is reduced. However even at sample sizes of three particles, considered to be of low significance by certain forensic services, ${ }^{31}$ some opportunity exists to exclude other possibilities even if no strong association can be made between a sample and one unique make of ammunition.

\subsection{Cartridges vs. hands}

Sample 10 was taken from the same ammunition as samples 1-6, however it was collected from a cartridge case. The separation between sample 10 and samples 1-6 supports previous observations that the GSR composition from hand samples may be different to that from cartridge cases, ${ }^{32}$ especially when a pistol has been used. Box plots of the elements (see ESI Fig. S2 $\dagger$ )
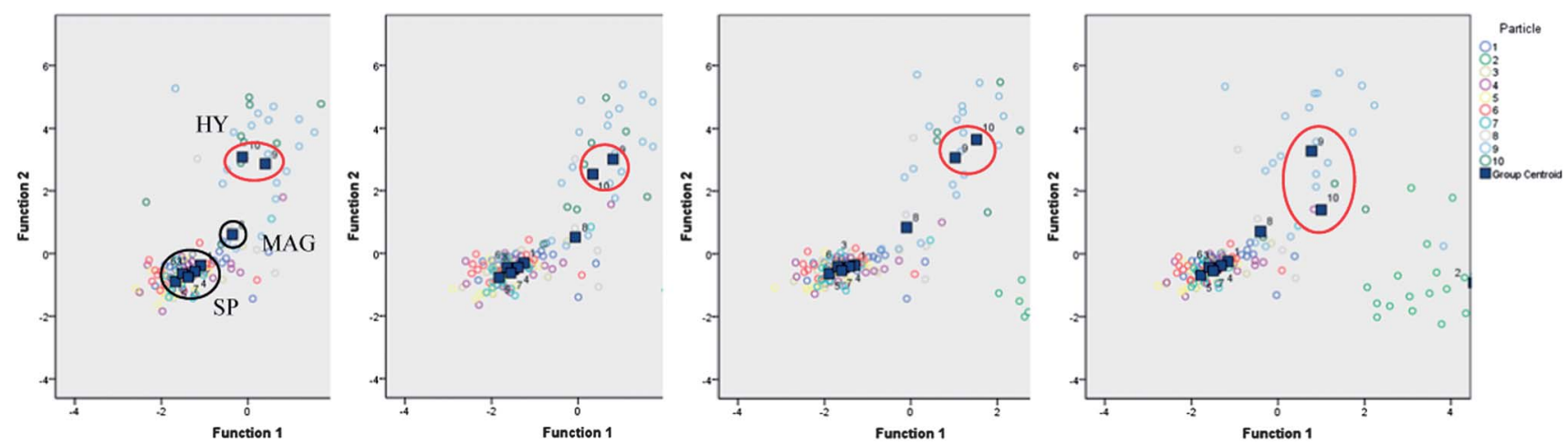

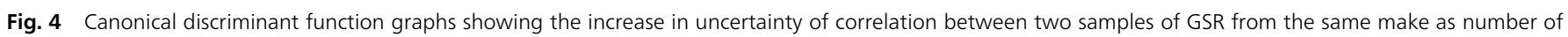
particles decrease from left to right. Individual graphs depict the results for 10,8,5 and 3 particles of sample 10 . 
show the reason for this separation. The cartridge case samples (sample 10) are low in $\mathrm{Ba}, \mathrm{Sb}$ and $\mathrm{S}$ compared with the samples from hands from the same make (samples 1-6). The cartridge case particles are significantly higher in $\mathrm{Cu}$ and $\mathrm{Zn}$ than the particles from hands. We have found (see ESI Fig. S2 $\uparrow$ ), that GSR taken from cartridge cases of different makes of Federal ammunition can also be separated using the protocol described in this work.

\section{Discussion}

The damage behavior is entirely expected: these GSR particles are largely metallic, and metals are largely immune to this sort of radiation damage. A $2.5 \mathrm{MeV}$ beam was used (instead of 3 $\mathrm{MeV}$ ) for convenience. In fact this is a rather more stringent test since the lower energy beam deposits about $10 \%$ more energy into the GSR particle. This particle is about $5 \mu \mathrm{m}$ in diameter, but the range of $3 \mathrm{MeV} \mathrm{H}$ into $\mathrm{BaO}_{2}$ is about $65 \mu \mathrm{m}$ : most of the beam energy is deposited in the carbon tape substrate.

Previous work has shown that the frequency of GSR particles of $1 \mu \mathrm{m}$ or less collected on hands is substantial, ${ }^{28,29}$ indicating a potential limitation of the technique. However, an ion beam with a smaller spot size would allow the detection of these smaller particles. Many such facilities exist already and several are under construction, ${ }^{30}$ with an expected spatial resolution down to $100 \mathrm{~nm}$ for analytical applications - an order of magnitude improvement on the beam used in this study.

In the relocation exercise, 9 out of 11 particles were located successfully. In this set of 11 GSR particles, three had sizes $\sim 1 \mu \mathrm{m}$. One of these was successfully found with a proton microbeam spot size of $1.5 \mu \mathrm{m}$. The other two could not be found at a time when the beam could not be focused better than $4 \mu \mathrm{m}$. Clearly, a proton microbeam which can be routinely focused below $1 \mu \mathrm{m}$ would be necessary for GSR work, considering the preponderance of small particles from the hands of living suspects.

The proton beam penetrates to the substrate (carbon tape in this case) and the EBS spectra and be accurately fitted, despite the geometrical complication that clearly exists, by a rather simple approximation of the target by a planar multi-layered target of varying proportions of heavy metals and carbon tape as a function of depth. This approximation will clearly bias the PIXE quantification depending on the details of the GSR geometry, and we therefore expect an accuracy of not better than $10 \%$.

The use of the relatively simple code OMDAQ ${ }^{24}$ instead of the much more accurate DataFurnace code used by Bailey and Jeynes $^{19}$ also impairs the precision of the method in the present work since it introduces yet further approximation. For the present purpose, this is of no consequence since the absolute precision does not significantly affect the multivariate analysis. The fact that reproducibility can be demonstrated shows that the precision is still at the $20 \%$ level or better, so that the approximations have only a limited effect on the quantitative results.

\section{Conclusions}

The new protocol presented and tested here is a demonstration of a streamlined analytical method for the quantitative analysis of GSR particles. Multivariate analysis confirms the validity of the approximations used in order to streamline the method, thus validating the analytical chemistry procedures used. This protocol has the ability to provide reproducible, quantitative measurements of a population of GSR particles. A quantitative discrimination protocol for GSR populations is unprecedented, and with it we have shown the grouping behavior of particles from three individual makes of cartridge from one manufacturer.

The method was shown to be non-destructive even under unrealistically long analysis times. Relocation of the particles from SEM images was demonstrated down to diameters of $1 \mu \mathrm{m}$, and new developments in the technology could lower this limit in the near future.

The reproducibility of the method for reanalysis of the same set of particles showed variations in the measured concentrations for most elements of around 10\%, reflecting spatial inhomogeneity in the particles as well as the precision of the protocol. Two populations of particles from the hands of the shooter of the same make of cartridge could not be discriminated under the protocol, while the discrimination between the 3 different makes of ammunition was obtained, the separation being significant past $99 \%$ confidence after analyzing the hands of shooters. This is particularly interesting in forensic science because we have studied three makes all containing lead, antimony and barium in the primer. Similarly, particles produced by one make of cartridge and different firearms were not discriminated.

The effect of sample size was also investigated and found to be significant at low particle numbers. However, evidential value might still be added even at these low numbers of particles by excluding possible sources for a GSR sample.

\section{Acknowledgements}

The authors would like to thank the EPSRC for funding the doctoral training grant (grant number EP/H500189/1) which funded Matthew Christopher's PhD, the EPSRC Knowledge Transfer Account and the EC Contract no. 227012 Support of Public and Industrial Research Using Ion Beam Technology (SPIRIT) for funding the ion beam time, LGC Forensics for provision of samples and the Surrey Police Tactical Firearms Unit, UK and the Metropolitan Police Force, UK for collection of samples.

\section{References}

1 G. M. Wolten and R. S. Nesbitt, On the Mechanism of Gunshot Residue Particle Formation, J. Forensic Sci., 1980, 25(3), 533-545.

2 O. Dalby, D. Butler and J. W. Birkett, Analysis of Gunshot Residue and Associated Materials-A Review, J. Forensic Sci., 2010, 55(4), 924-943.

3 A. Zeichner, B. Eldar, B. Glattstein, A. Koffman, T. Tamiri and D. Muller, Vacuum Collection of Gunpowder Residues from Clothing Worn by Shooting Suspects, and Their 
Analysis by GC/TEA, IMS, and GC/MS, J. Forensic Sci., 2003, 48(5), 961-972.

4 D. Laza, B. Nys, J. D. Kinder, A. K. D. Mesmaeker and C. Moucheron, Development of a quantitative LC-MS/MS method for the analysis of common propellant powder stabilizers in gunshot residue, J. Forensic Sci., 2007, 52(4), 842-850.

5 American Society for Testing and Materials, ASTM E158810e1: Standard Guide for Gunshot Residue Analysis by Scanning Electron Microscopy/Energy Dispersive X-ray Spectrometry, West Conshohocken PA, American Society for Testing and Materials, 2010.

6 F. S. Romolo and P. Margot, Identification of gunshot residue: a critical review, Forensic Sci. Int., 2001, 119(2), 195-211.

7 R. L. Singer, D. Davis and M. M. Houck, A survey of gunshot residue analysis methods, J. Forensic Sci., 1996, 41(2), 195198.

8 Z. Brozek-Mucha and A. Jankowicz, Evaluation of the possibility of differentiation between various types of ammunition by means of GSR examination with SEM-EDX method, Forensic Sci. Int., 2001, 123(1), 39-47.

9 Z. Brozek-Mucha, Chemical and Morphological Study of Gunshot Residue Persisting on the Shooter by Means of Scanning Electron Microscopy and Energy Dispersive X-Ray Spectrometry, Microsc. Microanal., 2011, 17(6), 972-982.

$10 \mathrm{~S}$. Shuttleworth and D. T. Kremser, Assessment of laser ablation and sector field inductively coupled plasma mass spectrometry for elemental analysis of solid samples, J. Anal. At. Spectrom., 1998, 13(8), 697-699.

11 Z. Abrego, A. Ugarte, N. Unceta, A. Fernandez-Isla, M. A. Goicolea and R. J. Barrio, Unambiguous Characterization of Gunshot Residue Particles Using Scanning Laser Ablation and Inductively Coupled PlasmaMass Spectrometry, Anal. Chem., 2012, 84(5), 24022409.

12 J. Coumbaros, K. P. Kirkbride, G. Klass and W. Skinner, Characterisation of 0.22 caliber rimfire gunshot residues by time-of-flight secondary ion mass spectrometry (TOFSIMS): a preliminary study, Forensic Sci. Int., 2001, 119(1), 72-81.

13 M. J. Bailey, C. Jeynes, B. J. Sealy, R. P. Webb and R. M. Gwilliam, On artefacts in the secondary ion mass spectrometry profiling of high fluence $\mathrm{H}(+)$ implants in GaAs, Nucl. Instrum. Methods Phys. Res., Sect. B, 2010, 268(11-12), 2051-2055.

14 J. Flynn, M. Stoilovic, C. Lennard, L. Prior and H. Kobus, Evaluation of X-ray microfluorescence spectrometry for the elemental analysis of firearm discharge residues, Forensic Sci. Int., 1998, 97(1), 21-36.

15 J. Bueno, V. Sikirzhytski and I. K. Lednev, Raman spectroscopic analysis of gunshot residue offering great potential for caliber differentiation, Anal. Chem., 2012, 84(10), 4334-4339.

16 S. Kage, K. Kudo, A. Kaizoji, J. Ryumoto, H. Ikeda and N. Ikeda, A simple method for detection of gunshot residue particles from hands, hair, face, and clothing using scanning electron microscopy/wavelength dispersive X-ray (SEM/WDX), J. Forensic Sci., 2001, 46(4), 830-834.

17 I. Nakai, Y. Ono, Q. H. Li, Y. Nishiwaki, K. Tanaka and S. Nakayama, Capability of a TES microcalorimeter SEMEDS system for forensic analysis of automotive paint and gunshot residue, Surf. Interface Anal., 2010, 42(5), 402-410.

18 M. J. Bailey, K. J. Kirkby and C. Jeynes, Trace element profiling of gunshot residues by PIXE and SEM-EDS: a feasibility study, X-Ray Spectrom., 2009, 38(3), 190-194.

19 M. J. Bailey and C. Jeynes, Characterisation of gunshot residue particles using self-consistent ion beam analysis, Nucl. Instrum. Methods Phys. Res., Sect. B, 2009, 267(12-13), 2265-2268.

20 F. S. Romolo, M. Christopher, M. Donghi, L. Ripani, C. Jeynes, R. Webb, N. Ward, K. Kirkby and M. Bailey, Integrated ion beam analysis (IBA) in gunshot residue (GSR) characterization, Forensic Sci. Int., submitted, http:// dx.doi.org/10.1016/j.forsciint.2013.05.006.

21 J. I. Goldstein, D. E. Newbury, P. Echlin, D. C. Joy, A. D. Romig, C. E. Lyman, C. Fiori and E. Lifshin, Scanning Electron Microscopy and X-ray Microanalysis, Plenum Press, New York, 2nd edn, 1992, pp. 472-492.

22 C. Jeynes, M. J. Bailey, N. J. Bright, M. E. Christopher, G. W. Grime, B. N. Jones, V. V. Palitsin and R. P. Webb, "Total IBA" - Where are We?, Nucl. Instrum. Methods Phys. Res., Sect. B, 2012, 271, 107-118.

23 C. Jeynes, N. P. Barradas, P. K. Marriott, G. Boudreault, M. Jenkin, E. Wendler and R. P. Webb, Elemental thin film depth profiles by ion beam analysis using simulated annealing - a new tool, J. Phys. D: Appl. Phys., 2003, 36(7), R97-R126.

24 G. W. Grime, The "Q-Factor" method: quantitative microPIXE analysis using RBS normalisation, Nucl. Instrum. Methods Phys. Res., Sect. B, 1996, 109-110, 170-174.

25 A. Zeichner and N. Levin, Collection efficiency of gunshot residue (GSR) particles from hair and hands using doubleside adhesive tape, J. Forensic Sci., 1993, 38, 571-584.

26 J. L. Campbell, N. I. Boyd, N. Grassi, P. Bonnick and J. A. Maxwell, The Guelph PIXE software package IV, Nucl. Instrum. Methods Phys. Res., Sect. B, 2010, 268(20), 3356-3363. 27 SPSS for Windows, Rel. 19.0.0, IBM SPSS Statistics, 2010.

28 S. Charles, B. Nys and N. Geusens, Primer composiiton and memory effect of weapons - some trends from a systematic approach in casework, Forensic Sci. Int., 2011, 212, 22-26.

29 Z. Brożek-Mucha, Distribution and properties of gunshot residue originating from a Luger $9 \mathrm{~mm}$ ammunition in the vicinity of the shooting gun, Forensic Sci. Int., 2009, 183(13), 33-44.

30 M. J. Merchant, G. W. Grime, K. J. Kirkby and R. Webb, A survey of two-stage focusing systems for nanobeam design, Nucl. Instrum. Methods Phys. Res., Sect. B, 2007, 260(1), 8-.

31 L. Reid, K. Chana, J. W. Bond, M. J. Almond and S. Black, Stubs Versus Swabs? A Comparison of Gunshot Residue Collection Techniques, J. Forensic Sci., 2010, 55(3), 753-756.

32 M. R. Rijnders, A. Stamouli and A. Bolck, Comparison of GSR Composition Occurring at Different Locations Around the Firing Position, J. Forensic Sci., 2010, 55(3), 616-623. 